Anestesiología

Abril-Junio 2022

Vol. 45. No. 2. pp 101-106

doi: $10.35366 / 103884$

\section{Evaluación de las características de la prescripción de opioides mayores otorgados por las farmacias de un Hospital General Latinoamericano}

\author{
Evaluation of the prescription characteristics of major opioids \\ dispensed by the pharmacies of a Latin American General Hospital
}

\author{
Dr. Alfredo Covarrubias-Gómez, ${ }^{*}$ Dr. Esteban Zavaleta-Monestel, ${ }^{\ddagger}$ \\ Dr. Jorge Arturo Villalobos-Madríz, ${ }^{\S}$ Dr. Roberto Rodríguez-Miranda, \\ Dr. José Pablo Díaz-Madríz," Dr. Bruno Serrano-Arias,",*** \\ Dra. Adriana Meoño-Elizondo, ${ }^{\ddagger}$ Dra. Wai Yee Zheng-Mok ${ }^{\S}$
}

Citar como: Covarrubias-Gómez A, Zavaleta-Monestel E, Villalobos-Madríz JA, Rodríguez-Miranda R, Díaz-Madríz JP, Serrano-Arias B, et al. Evaluación de las características de la prescripción de opioides mayores otorgados por las farmacias de un Hospital General Latinoamericano. Rev Mex Anestesiol. 2022; 45 (2): 101-106. https://dx.doi.org/10.35366/103884

RESUMEN. Introducción: Los opioides se han utilizado para el alivio del dolor en diversos contextos. Su uso puede favorecer efectos indeseables. Actualmente los países industrializados viven una crisis de opioides. Objetivos: Caracterizar los opioides mayores despachados en términos de especialidad médica y de dosis total en miligramos equivalentes a morfina (MME) en un Hospital General Latinoamericano. Material y métodos: Se realizó un estudio retrospectivo y observacional de prescripciones de opioides mayores despachadas en las Farmacias de un Hospital General Latinoamericano entre el año 2017 y 2020. Se tomó información de la base de datos del Ministerio de Salud. Se excluyeron las recetas que contenían estupefacientes no opioides y fentanilo inyectable. Se realizó una estandarización a MME para analizar la distribución de la prescripción según médicos y pacientes. Resultados: La cantidad total de recetas despachadas correspondientes a opioides mayores fue de 5,366 prescripciones. La morfina inyectable fue el medicamento más prescrito (46\%) y como principio activo obtuvo $54 \%$ de todas las recetas estudiadas (considerando todas las vías de administración). Medicina general prescribió la mayoría de las recetas con un $48 \%$, lo que representó $42 \%$ del total de MME. Conclusiones: La morfina es el principio activo preferido por los médicos evaluados en este estudio, la vía de administración parenteral es la más utilizada. Medicina general es el área que más prescribe opioides. Es pertinente crear políticas educativas en materia de opioides; por ello, resulta conveniente caracterizar la prescripción de manera cualitativa considerando información como el diagnóstico y las dosis diarias utilizadas.

ABSTRACT. Introduction: Opioids have been used for pain relief in various settings. Its use can promote undesirable effects. Industrialized countries are currently experiencing an opioid crisis. Objective: To characterize the prescription of major opioids considering the medical area that made the prescription and total dose in milligrams morphine equivalents (MME) at a general hospital in Latin America. Material and methods: A retrospective and observational study was carried out. The number of prescriptions for major opioids dispensed in the pharmacies of a general hospital in Latin America, between 2017 and 2020, were documented. Information was taken from the database of the Ministry of Health. Prescriptions containing non-opioid narcotic drugs and injectable Fentanyl were excluded. A standardization to MME was carried out to analyze the distribution of prescriptions. Results: The total number of prescriptions filled for major opioids was 5,366 prescriptions. Injectable morphine was the most prescribed drug (46\%) and as an active principle it obtained $54 \%$ of all the prescriptions studied (considering all routes of administration). General medicine prescribed most prescriptions (48\%), which represented $42 \%$ of the total MME. Conclusions: Morphine is the substance preferred by the doctors evaluated in this study, the parenteral route of administration is the most used. General medicine is the area that prescribes most of the opioids. It is pertinent to create educational policies on opioids; therefore, it is convenient to characterize the prescription qualitatively considering information such as the diagnosis and the daily doses used.

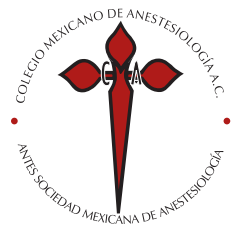

Palabras clave: Analgésicos, opioides, prescripción, hospital, medicina.

Keywords:

Painkillers, opioids, prescription, hospital, medicine.

* Departamento de Educación en Investigación en el Centro Algia para la Educación en Salud, Departamento de Medicina del Dolor y Paliativa en el Instituto Nacional de Ciencias Médicas y Nutrición «Salvador Zubirán», Ciudad de México en México. ‡ Departamento de Farmacia en el Hospital Clínica Bíblica, San José en Costa Rica.

$\S$ Departamento de Farmacia de la Universidad de Ciencias Médicas (UCIMED), San José en Costa Rica. " Departamento de Anestesiología. Hospital Clínica Bíblica,

San José, Costa Rica.

"Departamento de Farmacia en el Hospital Clínica Bíblica, San José, Costa Rica.

** Departamento de Farmacología de la Universidad de Ciencias Médicas (UCIMED), San José en Costa Rica.

Correspondencia:

Alfredo Covarrubias-Gómez Departamento de Medicina del Dolor y Paliativa en el Instituto Nacional de Ciencias Médicas y Nutrición «Salvador Zubirán», Ciudad de México en México. Vasco de Quiroga Núm. 15, Col. Toriello Guerra Sección XVI Alcaldía Tlalpan, 14000, Ciudad de México, México. E-mail: alfredo.covarrubias@ algia.org.mx

Recibido: 10-12-2021

Aceptado: 21-01-2022 


\section{INTRODUCCIÓN}

T os opioides son conocidos como compuestos que tienen capacidad de amortiguar y disminuir el dolor por medio de la unión con receptores específicos encontrados en el sistema nervioso, estos compuestos son derivados de la planta Papaver somniferum o amapola ${ }^{(1)}$.

En los últimos 10 años ha venido el apogeo de nuevas moléculas opioides sintéticas como análogos del fentanilo y otros disponibles en Costa Rica como la buprenorfina, las cuáles son moléculas con mecanismos de acción más selectivos disminuyendo los efectos adversos y aumentando la potencia farmacológica ${ }^{(2)}$.

Este grupo de fármacos se utiliza comúnmente para el tratamiento de dolores fuertes e intensos (en escala verbal análoga calificado como severo o insoportable), dando como resultado en la mayoría de los casos una analgesia efectiva. Sin embargo, es común ver efectos adversos que son en su mayoría dependientes de la dosis como náusea, euforia y depresión respiratoria. Otros efectos no dependientes de la dosis como el estreñimiento también pueden ser identificados con frecuencia ${ }^{(1)}$.

Los opioides se subdividen en opioides mayores (morfina, oxicodona, fentanilo, metadona, buprenorfina) y menores (codeína, tramadol, dihidrocodeína) ${ }^{(1)}$. Los opioides mayores, a diferencia de los opioides menores, generalmente carecen de techo terapéutico y las dosis analgésicas pueden crecer de forma significativa en algunos casos. La dosis analgésica ideal es aquella que alivia el dolor sin que se presenten efectos adversos. En el contexto del alivio del dolor crónico nooncológico su empleo se ha asociado a farmacodependencia y adicción en países desarrollados ${ }^{(1)}$.

El aumento significativo en la accesibilidad de los medicamentos opioides prescritos en el nuevo milenio impulsó una creencia a que los analgésicos opioides eran «seguros» y permitió una supervisión insuficiente o ineficaz de los mismos, y a medida que el uso médico general se hizo más común, también lo hizo el uso no médico ${ }^{(3)}$.

Esta epidemia se ha desarrollado desde dos posiciones. Por un lado, el uso de analgésicos opioides prescritos (que aproximadamente representan la mitad de las muertes por sobredosis); y por otro lado, el creciente número de medicamentos que se adquieren de manera ilícita ${ }^{(4)}$. Esta relación llevó a aproximadamente 25 millones de estadounidenses al uso no médico de opioides ${ }^{(5)}$.

El consumo de opioides ha logrado niveles epidémicos en los Estados Unidos con un incremento de 200\% en las muertes por sobredosis con la utilización de medicamentos opioides entre el año 2000 y 2014(6), llegando a su punto máximo en 2010, siendo el undécimo año consecutivo en el que se registró un aumento en la cantidad de personas fallecidas relacionadas con los opioides ${ }^{(7)}$.
Las estadísticas son preocupantes ya que algunos estudios describen que entre 90-150 estadounidenses mueren por sobredosis de opioides cada día ${ }^{(8,9)}$. Los centros para el control y la prevención de enfermedades (CDC por sus siglas en inglés) de los Estados Unidos, estimaron que en 2016 ocurrieron más de 60,000 muertes por sobredosis, generando desde entonces un aumento dramático del doble de muertes debido a los opioides sintéticos ${ }^{(10)}$.

Ante la crisis de salud pública en los Estados Unidos se han promovido distintas políticas como un abordaje en la prescripción de medicamentos opioides. Se han establecido programas de monitoreo de medicamentos recetados en una base de datos electrónica que reúne información sobre el historial de medicamentos prescritos a cada paciente ${ }^{(4)}$.

La Comisión Presidencial de Lucha contra las Toxicomanías y la Crisis de Opioides hizo una declaración nacional de emergencia en julio de 2017, para manifestar su situación de sobredosis de opioides como un problema grave a nivel nacional $^{(4,11,12)}$. Ante esta crisis se han decretado varias políticas para abordar la promoción de opioides y restringir las oportunidades de uso ilícito ${ }^{(13)}$.

En Costa Rica, a partir del año 2003, el Ministerio de Salud ha reportado un aumento sustancial del uso de opioides en el sector público y privado (específicamente en la utilización de morfina) ${ }^{(14)}$. El Instituto sobre Alcoholismo y Farmacodependencia (IAFA de Costa Rica) ha realizado diferentes investigaciones al respecto en jóvenes sobre el consumo de drogas ilícitas y el manejo paliativo no adecuado en el paciente oncológico $^{(15-17)}$.

En el año 2018, el IAFA detectó que el consumo más alto de medicamentos sin prescripción médica en estudiantes de secundaria fueron los opioides, estando éstos, por encima de los medicamentos estimulantes y otros sedantes ${ }^{(17)}$. Por su parte, el Instituto Costarricense de Drogas (ICD) en un reporte histórico sobre el decomiso de drogas ilícitas declaró una disminución de embargos de opioides, principalmente de la heroína, en la última década. Esto se atribuye al acceso a mayor variedad de opioides en el mercado ilícito ${ }^{(18)}$. A pesar de lo anterior, históricamente las tasas de consumo de opioides en Costa Rica son bajas ${ }^{(19)}$.

En noviembre de 2016, el Ministerio de Salud declaró oficialmente el uso obligatorio de la receta digital para todos los profesionales que se encuentren involucrados con el uso y manejo de medicamentos psicotrópicos y estupefacientes. Lo anterior, con el fin de mejorar el control de la prescripción, despacho y la comercialización de estos medicamentos, para así evitar su mal uso y farmacodependencia ${ }^{(14)}$.

Los médicos y odontólogos son los únicos autorizados para la prescripción de medicamentos psicotrópicos y estupefacientes; los farmacéuticos son los únicos autorizados para su despacho ${ }^{(14)}$. Con el uso de este sistema, el Ministerio de Salud obtiene registro de todos los profesionales y pacientes 
involucrados en el manejo del medicamento, haciendo posible la trazabilidad en caso de alguna sospecha o error ${ }^{(20)}$.

Este estudio tiene como objetivo caracterizar y examinar la distribución y patrones de la prescripción de opioides en un Hospital General Latinoamericano (Hospital Clínica Bíblica, San José, Costa Rica).

\section{MATERIAL Y MÉTODOS}

Se obtuvieron un total de 20,390 recetas de estupefacientes, en un período comprendido entre enero 2017 hasta el tercer trimestre del año 2020. Se incluyeron recetas despachadas pertenecientes a tres de las farmacias que tiene el Hospital Clínica Bíblica.

La información fue obtenida de la base de datos del Ministerio de Salud de Costa Rica y estos incluían un reporte detallado con: fecha de la receta, número de receta, orden de compra, droguería, ingresos, saldos, nombre completo y código profesional del médico, principio activo, unidades despachadas, nombre y número de identificación del paciente; cada documento estaba dividido por localidad y año respectivo.

Con estos datos se creó un archivo en Excel, donde se incluyó únicamente fecha de la receta, código del médico, con lo que se pudo obtener por medio de la página en línea del Colegio de Médicos y Cirujanos de Costa Rica la especialización de cada uno de ellos, también se usaron las unidades despachadas, cédula del paciente y principio activo.

En el análisis se utilizaron criterios de exclusión, siendo estos médicos cuya especialidad no aparezca en la página del Colegio de Médicos y Cirujanos, prescripciones por fentanilo inyectable, ya que su manejo es únicamente intrahospitalario y no en consulta externa. Los médicos que tuvieran registradas dos especializaciones o más, se unificaron en el grupo de su última especialidad o bien si la primera era necesaria para la obtención de la siguiente especialidad.

Para los opioides se contabilizaron un total de 5,366 recetas de opioides incluyendo como principio activo: morfina con concentraciones de $2 \mathrm{mg} / \mathrm{mL}, 15 \mathrm{mg} / \mathrm{mL}, 20 \mathrm{mg}, 30 \mathrm{mg}$, fentanilo de 25,50 y $100 \mu \mathrm{g}$ (parches), metadona de 5 y 10 $\mathrm{mg} / 2 \mathrm{~mL}$, tapentadol de 50 y $100 \mathrm{mg}$, oxicodona de 10, 20 y $40 \mathrm{mg}$ y buprenorfina parches de 20, 30 y $40 \mathrm{mg}$.

Se estimaron dos cantidades principales de interés para cada medicamento: dosis total del principio activo por cada paciente y por cada prescriptor. Por su parte, se realizó una estandarización a equivalentes de miligramos de morfina (MME por sus siglas en inglés) utilizando factores de conversión específicos del Código Nacional de Medicamentos, esto con el fin de poder comparar la prescripción total de opioides sin importar el principio activo ${ }^{(20)}$.

La dosis en MME se calculó con la concentración de cada principio activo por el factor de conversión de los opioides y este resultado fue multiplicado nuevamente por la cantidad de unidades despachadas a cada paciente, para obtener la dosis total.

\section{RESULTADOS}

La cantidad total de recetas despachadas fue de 5,366 prescritas por 648 médicos para una totalidad de 2,265 pacientes. De las recetas relacionadas con opioides se prescribió un total de 5,095,685 MME (en el período de tiempo estudiado).

La prescripción según los principios activos y fórmula farmacéutica se distribuyó como se observa en la Figura 1. La morfina inyectable es el medicamento que más se prescribió con un total de 2,478 recetas, representando un $46 \%$ del total de prescripciones. Ahora bien, la morfina, como principio activo (independientemente de la vía de administración) representó un 54\% de todas las recetas estudiadas.

Hubo un total de 46 diferentes especialidades que prescribieron estupefacientes en el hospital, los más frecuentes se muestran en la Figura 2. Al tomar en cuenta las especialidades con mayor número de prescripciones se obtuvo que medicina general prescribió el mayor número de las recetas, representando un 48\% de la totalidad y un 42\% del MME.
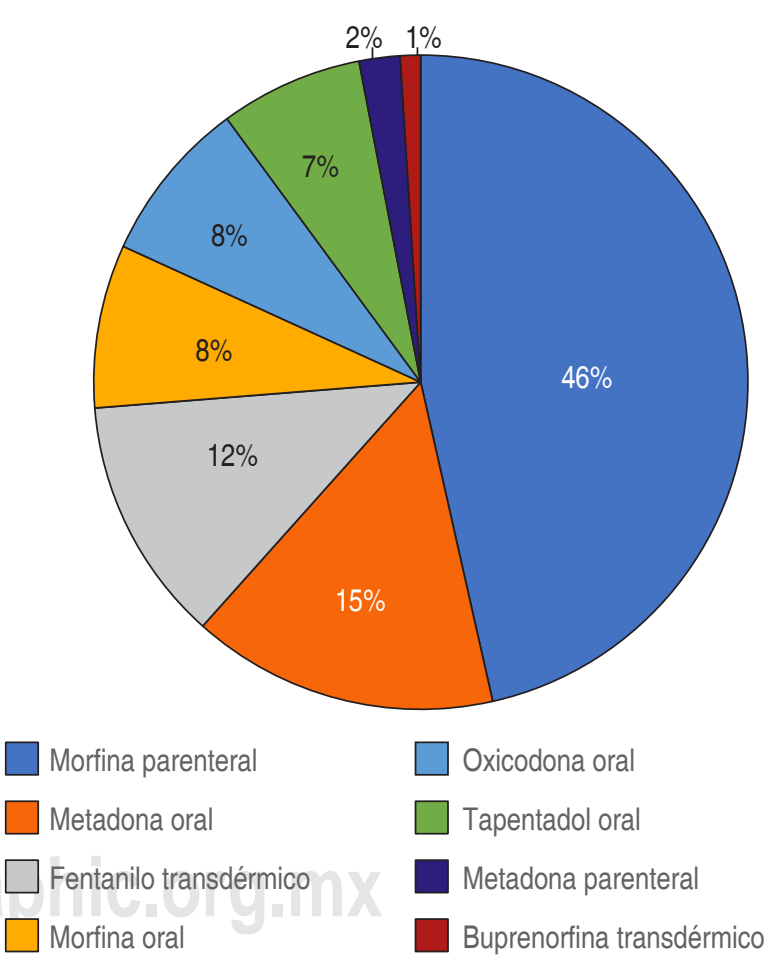

Figura 1: Distribución de prescripciones según principio activo y vía de administración.

Se observa la distribución de la prescripción de acuerdo con el principio activo. La morfina es el fármaco más empleado. Podemos identificar que la morfina parenteral ocupa $46 \%$ de las prescripciones, mientras que su formulación oral sólo abarca $8 \%$ de las prescripciones. 


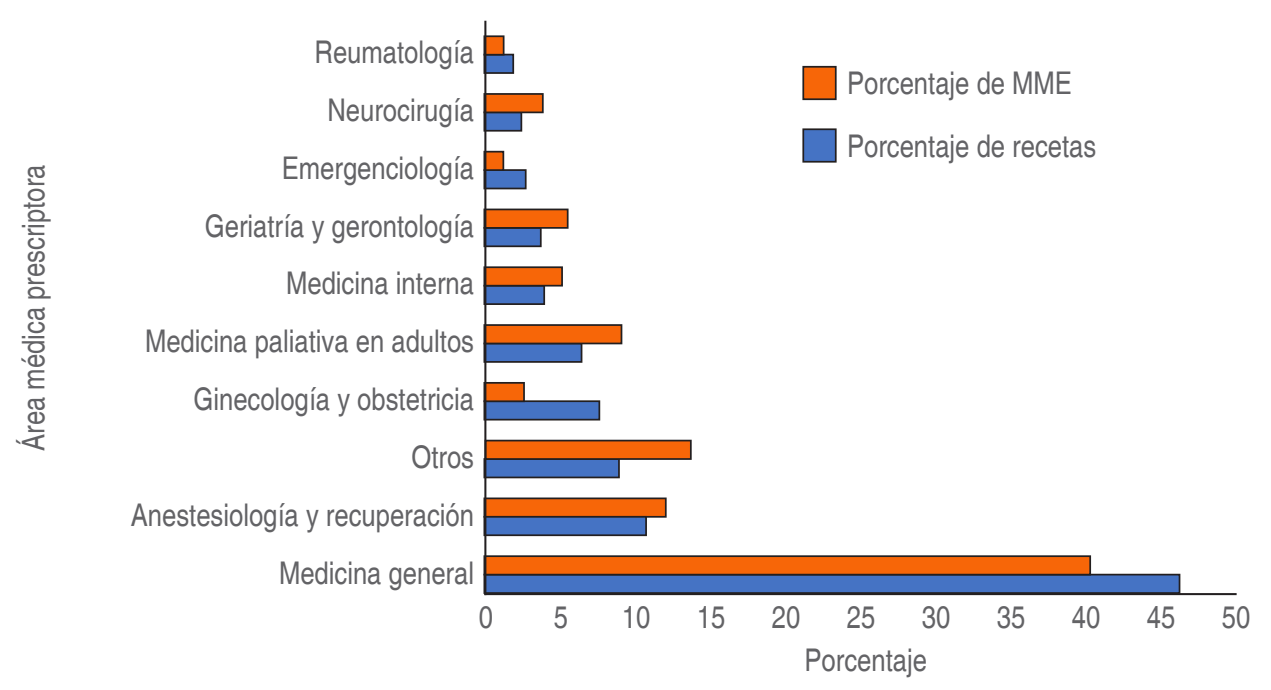

Figura 2:

Porcentaje de cantidad total
de recetas y miligramos
equivalentes a morfina según
el área médica prescriptora.
Se observan los porcentajes
correspondientes a la cantidad total
de recetas prescritas y los miligramos
equivalentes a morfina (MME) recetados.
Las columnas se agrupan de acuerdo al
área médica que realizó la prescripción.
Los médicos generalistas son el área que
más recetas otorga y más MME utiliza.
MME = miligramos equivalentes a morfina.

Relacionando los valores paciente-receta, del total de 2,265 pacientes, el que tuvo la mayor cantidad de recetas alcanzó 92. Al analizar los percentiles resultó que $10 \%$ superior (227 pacientes) obtuvo 48\% (2,599 recetas) de la totalidad, evidenciando que el restante $52 \%$ (2,767 recetas) se distribuye en los demás percentiles de manera minoritaria (Figura 3).

En la relación médico-receta, el mayor número de recetas realizadas por un solo médico fue de 387, representando un $7 \%$ de todas las recetas recolectadas. Como se observa en la Figura 4, 10\% superior de los médicos incluidos (65) representan 68\% del porcentaje de MME total. Además, el valor individual de médico-MME más alto resultó en 188,525 MME, representando un 4\% de las dosis totales de MME.

\section{DISCUSIÓN}

Actualmente, la situación de Costa Rica con respecto a la adicción de opioides es de un porcentaje bajo con una dosis diaria definida de opioides con fines estadísticos (DDD-S) de siete en comparación con países centroamericanos donde no se sobrepasan las 30 DDD-S, esto según el reporte de uso de narcóticos de las Naciones Unidas en el período 2016-2018 ${ }^{(21)}$.

Al contrastar este número con países como Estados Unidos, Reino Unido o Canadá que cuentan con DDD-S que rondan los 1,500 se observa el poco uso relativo de estas sustancias en Costa Rica(21) ${ }^{(21}$ Esto sumado al control estricto que se implementó con las recetas especiales para evitar la prescripción indebida y su mal uso ${ }^{(17,19)}$.

Al finalizar la Figura 1, queda claro que el uso de morfina vía oral y parenteral es el que predomina en la población estudiada. Este medicamento es de elección en una variedad de indicaciones, principalmente el dolor agudo, postoperatorio u oncológico ${ }^{(22)}$.
Llama la atención que la vía de administración preferida para la morfina fue la parenteral, a pesar de que se evaluaron pacientes en el ámbito ambulatorio. Un dato importante para resaltar es que a pesar de que la buprenorfina en parches es una de las opciones terapéuticas preferidas actualmente, su ingreso al mercado fue a partir de abril de 2020, por lo que no representa un porcentaje significativo de las prescripciones en el período estudiado.

Al correlacionar estos datos con los resultados mostrados en la Figura 2, naturalmente las especialidades médicas en los primeros puestos según la cantidad de prescripciones coinciden con las indicaciones aprobadas para los opioides, ya que en los primeros lugares se encuentran medicina paliativa, ginecología u obstetricia, anestesiología y recuperación, los cuales prescriben medicamentos para el manejo del dolor intenso, como por ejemplo postoperatorio ${ }^{(22)}$. Curiosamente ni cirugía general ni ortopedia forman parte de los primeros puestos en prescripción de opioides cuando históricamente entran en ese grupo.

En Costa Rica, sólo los médicos con especialidades cuentan con la autorización de prescribir opioides mayores a pacientes que lo requieran y por un tiempo razonable. Sin embargo, esta no es la realidad que se refleja en los datos encontrados a nivel privado, siendo los médicos generales los que presentan más prescripciones de opioides mayores que cualquier otra especialidad médica.

Esto se vuelve a confirmar al observar la Figura 2, en donde se muestra que medicina general abarca un gran porcentaje tanto de cantidad de prescripciones como el total de MME, todo esto a pesar de que el hospital cuenta con un 95\% de su personal médico que es especialista y un 5\% que son generalistas.

Es importante resaltar que al ser los médicos generales los responsables de la gran mayoría de este tipo de pres- 
cripciones, es pertinente desarrollar estrategias educativas a nivel nacional para promover el uso racional de estupefacientes y evitar crear una cultura de sobreprescripción en los años venideros.

Otro posible factor influyente en lo anterior es que el hospital en cuestión es un centro médico referente en el ámbito nacional, además, es un hospital general en donde se admiten pacientes médicos, críticos, quirúrgicos y se da el servicio de consulta externa. Por otro lado, el centro de salud se localiza en una zona céntrica con consultorios particulares a sus alrededores y al contar con el suficiente abastecimiento de este tipo de drogas, es lugar de preferencia de prescriptores externos.

$\mathrm{Al}$ analizar las Figuras 3 y 4, se nota una distribución desproporcionada entre los percentiles de pacientes contra cantidad de recetas y cantidad de MME según prescriptores. Comparando estos resultados con datos del ámbito internacional, se observa una similitud en la poca homogeneidad de prescripción, en Estados Unidos, 1\% de los médicos prescriben un $49 \%$ de las dosis totales de opioides registradas, mientras que en nuestro país, el percentil 10 de los pacientes estudiados abarcan $48 \%$ de las recetas despachadas y el percentil 10 de prescriptores recetan $68 \%$ del $\mathrm{MME}^{(21)}$.

Estudios estadounidenses concluyen que se deben enfocar estrategias educativas e intervenciones en ese grupo de médicos que en su mayoría son de la rama de medicina familiar y medicina interna, en cambio en Costa Rica predominan medicina general y especialidades quirúrgicas ${ }^{(21)}$.

Esta investigación tuvo varias limitaciones, los datos fueron obtenidos únicamente del Hospital Clínica Bíblica

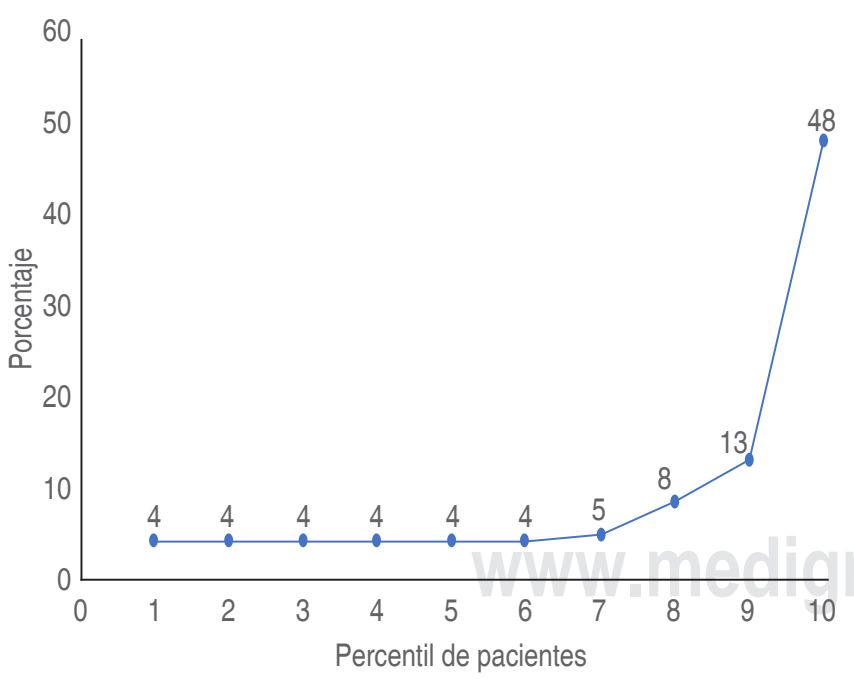

Figura 3: Porcentaje de recetas según percentil de pacientes. Se identifica el porcentaje de recetas según el percentil de pacientes. Al analizar los percentiles resultó que 10\% superior (227 pacientes) obtuvo 48\% (2,599 recetas) de la totalidad, evidenciando que el restante $52 \%$ (2,767 recetas) se distribuye en los demás percentiles de manera minoritaria.

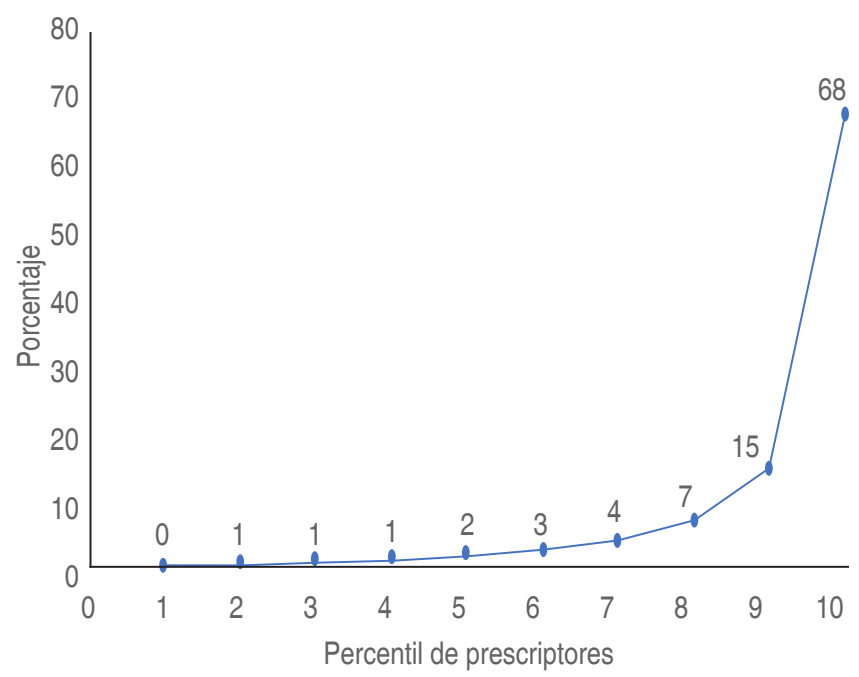

Figura 4: Porcentaje del miligramos equivalentes a morfina según percentil de prescriptores.

Se identifica el porcentaje de miligramos equivalentes a morfina (MME) según el percentil de prescriptores, $10 \%$ superior de los médicos incluidos (65) representan $68 \%$ del porcentaje de MME total. Además, el valor individual de médico-MME más alto resultó en 188,525 MME, representando un $4 \%$ de las dosis totales de MME. $\mathrm{MME}=$ miligramos equivalentes a morfina.

cuyo centro es privado y la muestra no es representativa de la realidad nacional. La información extraída no incluyó información sustancial, como edad y sexo del paciente, dosis diaria, relación médico-paciente, adherencia al tratamiento ni diagnóstico, por lo que no se caracterizó la prescripción de opioides de manera cualitativa.

\section{CONCLUSIONES}

La morfina en general es el principio activo más prescrito por los médicos incluidos en el estudio debido a su amplia variedad de indicaciones y versatilidad de administración, y que, a pesar de ser un ámbito mayormente ambulatorio, la vía de administración parenteral es la predilecta. Existe en general una distribución heterogénea del patrón prescripción, ya que en una minoría de pacientes y de médicos se concentra la mayoría de las prescripciones de opioides, reflejándose las características de servicio y localización del hospital.

Medicina general es el área que más prescribe opioides. Por lo que es pertinente crear políticas educativas para garantizar la prescripción racional e informada. Por esto, es importante caracterizar la prescripción de manera cualitativa a partir de información como diagnósticos y dosis diarias definidas, con el fin de detectar puntos de mejora con respecto a esta situación en el ámbito nacional.

Conflicto de intereses: Los autores no presentan conflicto de intereses. 


\section{REFERENCIAS}

1. Duce TS, Rollán AB, López ME, Camarasa PJ. Terapéutica en Atención Primaria. Uso de los opioides en pacientes con dolor oncológico. SEMERGEN Soc Esp Med Rural GenEd Impr. 2007;33:520-528.

2. Álvarez Y, Farré M. Farmacología de los opioides. Adicciones. 2005; 17:21-40.

3. Wilkerson RG, Kim HK, Windsor TA, Mareiniss DP. The opioid epidemic in the United States. Emerg Med Clin North Am. 2016;34:e1-23.

4. Rutkow L, Vernick JS. Emergency legal authority and the opioid crisis. N Engl J Med. 2017;377:2512-2514.

5. Center for Behavioral Health Statistics and Quality. Results from the 2011 NSDUH: Summary of National Findings, SAMHSA, CBHSQ [Internet]. Substance Abuse and Mental Health Services Administration. 2012 [cited 29 Mayo, 2021]. Available in: https://www.samhsa.gov/ data/sites/default/files/Revised2k11NSDUHSummNatFindings/ Revised2k11NSDUHSummNatFindings/NSDUHresults2011.htm

6. Rudd RA, Aleshire N, Zibbell JE, Gladden RM. Increases in drug and opioid overdose deaths-United States, 2000-2014. Morb Mortal Wkly Rep. 2016;64(50-51):1378-1382.

7. Murphy S, Kochanek KD, Xu J, Heron M. Deaths: final data for 2012 Natl Vital Stat Rep. 2015;63:1-117.

8. Rudd RA, Seth P, David F, Scholl L. Increases in drug and opioidinvolved overdose deaths-United States, 2010-2015. Morb Mortal Wkly Rep. 2016;65(50-51):1445-1452.

9. Shulman J, Roodenburg E, DiMaria R. The opioid epidemic: spotlighting international efforts to address the crisis [Internet]. KPMG; 2018. Available in: https://assets.kpmg/content/dam/kpmg/ca/pdf/2018/04/ the-opioid-epidemic.pdf

10. Hedegaard H, Warner M, Miniño AM. Drug overdose deaths in the United States, 1999-2015. National Center for Health Statistics (USA), editor. 2017; Available in: https://stacks.cdc.gov/view/cdc/44356

11. Christie C, Baker C, Cooper R, Kennedy PJ, Madras B, Bondi P. The president's commission on combating drug addiction and the opioid crisis. Wash DC US Gov Print Off. 2017.

12. Gostin LO, Hodge JG Jr, Noe SA. Reframing the opioid epidemic as a national emergency. JAMA. 2017;318(16):1539-1540.

13. Rutkow L, Vernick JS, Alexander GC. More states should regulate pain management clinics to promote public health. Am J Public Health. 2017;107(2):240-243.
14. Instituto sobre Alcoholismo y Farmacodependencia. VI Encuesta Nacional sobre Consumo de Drogas en Población General 2015 [Internet]. IAFA; 2018. Disponible en: https://www.iafa.go.cr/images/ descargables/investigaciones/Encuesta--Nacional-de-Drogas-2015.pdf

15. Sandí BS, Sandí EL. Dependencia a opioides y su tratamiento. Rev Clínica Esc Med UCR-HSJD [Internet]. 2016;6(1). Disponible en: https://revistas.ucr.ac.cr/index.php/clinica/article/view/23063

16. Vargas BA. Manejo no adecuado del dolor por cáncer en Costa Rica: ¿Un problema de formación académica? Acta Médica Costarricense [Internet]. el 22 de julio de 2020 [citado el 29 de mayo de 2021];56(2). Disponible en: http://actamedica.medicos.cr/index.php/Acta_Medica/article/view/835

17. Arce BA, Chacón SW, Dobles UA, Méndez MJ, Ramírez AY, Salas AC. $\mathrm{V}$ encuesta nacional sobre consumo de sustancias psicoactivas en población de educación secundaria 2018. Instituto sobre Alcoholismo y Farmacodependencia. 2019, 80.

18. Unidad de Información y Estadística Nacional sobre Drogas. Boletín Estadístico Enero 2021 [Internet]. ICD; 2021. Disponible en: https:// www.icd.go.cr/portalicd/images/docs/uid/boletin_esta_uid/BE_2021/ BE_UID_Ene-21.pdf

19. Valverde Alfaro E, Arce Von Herold A. Caracterización demográfica de los pacientes con trastorno por uso de sustancias egresados del Hospital Nacional Psiquiátrico en el año 2011: Propuesta de terapia grupal de 4 sesiones en el manejo de consumo de drogas en pacientes que además padecen esquizofrenia o trastornos afectivos que estén hospitalizados en el Hospital Nacional Psiquiátrico [Tesis de postgrado]. San José: Universidad de Costa Rica; 2013.

20. Ministerio de Salud Costa Rica. Salud oficializa y activa Receta Digital [Internet]. Ministerio de Salud Costa Rica. 2017 [citado el 29 de mayo de 2021]. Disponible en: https://www.ministeriodesalud.go.cr/index. php/noticias/noticias-2017/1130-salud-oficializa-y-activa-receta-digital

21. International Narcotics Control Board. Narcotic Drugs: Estimated World Requirements for 2020 [Internet]. Austria: United Nations; 2020 p. 220-265. Available in: https://www.incb.org/documents/NarcoticDrugs/Technical-Publications/2019/Narcotic_Drugs_Technical_ Publication_2019_web.pdf

22. Dowell D, Haegerich T, Chou R. CDC Guideline for prescribing opioids for chronic pain-United States, 2016. MMWR Recomm Rep [Internet]. 2016 [Cited 29 May 2021]; 65. Available in: https://www.cdc.gov/mmwr/ volumes/65/rr/rr6501e1.htm 